ECCOMAS

\section{Proceedia}

\title{
DISCRETE MODEL FOR OUT-OF-PLANE LOADED RANDOM MASONRY
}

\author{
D. Baraldi ${ }^{1}$, Emanuele Reccia ${ }^{2}$ and A. Cecchi $^{3}$ \\ Università IUAV di Venezia - DACC, Department of Architecture, Construction, Conservation \\ Terese, Dorsoduro 2206, 30123, Venice (Italy) \\ ${ }^{1}$ e-mail: danielebaraldi@iuav.it \\ 2 e-mail: emreccia@iuav.it \\ 3 e-mail: cecchi@iuav.it
}

Keywords: Discrete rigid block model, random masonry, homogenization, modal analysis.

\begin{abstract}
In this contribution, a simple and effective discrete element model based on rigid blocks and elastic interfaces with fixed contact topology, originally introduced for modeling regular masonry panels, is extended to the case of random masonry by introducing a perturbation parameter able to vary the width of each block. The proposed model is then able to better reproduce the microstructural behavior of historical masonry, that is characterized by dry or weak mortar joints between strong blocks, and, in particular, that is characterized by blocks often arranged irregularly.

The hypothesis of rigid blocks, together with fixed contact topology between blocks due to the small displacements assumption, allows adopting an efficient solution method based on the determination of the stiffness matrix of the masonry assemblage. In this case, the stiffness matrix is able to account for the irregular block arrangement and, similarly to the case of regular masonry, the stiffness matrix is based on local joint stiffness, given that the contact actions along the joints are function of the relative displacements between adjacent blocks and the corresponding interface stiffness.

Several numerical tests varying the random perturbation parameter are performed in order to evaluate the influence of randomness on masonry specimen behavior with respect to the regular case. Particular attention is given to the dynamic field by performing out-of-plane modal analysis of masonry panels.

Furthermore, a homogenization procedure is applied to the random masonry and a numerical evaluation of the scatter between the discrete models and a $2 D$ Reissner-Mindlin plate model is performed for varying perturbation parameter and for increasing heterogeneity parameter. As expected, when the number of heterogeneities in the structure is large enough, the average response of the random discrete model converges to an asymptotic response.
\end{abstract}




\section{INTRODUCTION}

As well known in the community of architects and civil engineers, the assessment of masonry structural behavior is of particular interest, given that masonry constructions are one of the most widespread type of traditional building in Europe and in Italy in particular. Moreover, recurring seismic events represent one of the main causes of collapse for this category of buildings, with in- and out-of-plane collapse mechanisms that need to be evaluated and prevented. The literature dedicated to the assessment of masonry at different scale levels by means of analytic and numeric models is huge [1,2]. On one hand, refined models are able to investigate the local behavior of masonry at a small scale level, but modeling and computational difficulties may arise when entire buildings or building portions need to be studied. On the other hand, continuous models are able to study masonry structures as a whole, accounting for average material mechanical properties based on single constituent characteristics, but they are unable to evaluate local behavior governed by material microstructure or by architectural details.

Considering historical masonry, real case studies are often characterized by walls having blocks arranged irregularly. The literature dedicated to this aspect is present [3-8], but most of the existing analytical and numerical models are dedicated to the case of regular masonry. Refined numerical models such as the discrete ones [9] are able to study the behavior of independent (distinct) elements in contact with several neighbors, then they are able to represent the mechanical properties of historical masonry, that is characterized by weak and small joints with respect to strong and well-sized blocks, allowing to assume that damage occurs more frequently along joints. Furthermore, random arrangement can be taken into account without varying significantly the complexity of the discrete system and the consequent computational effort of the numerical analysis.

Here, a simple and effective discrete model, originally introduced for studying regular masonry in the elastic field subject to in- and out-of-plane loads [10], already extended to the field of material nonlinearity [11], is extended to the case of random or quasi-periodic masonry by introducing a perturbation parameter able to vary the width of each block. For this purpose, the work of one of the authors [6] is considered and improved, given that the hypothesis of fixed contact topology between blocks, together with the hypothesis small displacements, allow to adopt an efficient solution method based on the determination of the stiffness matrix of the masonry assemblage, that is extended to the case of random masonry.. Attention is devoted to the out-of-plane behavior, given that out-of-plane vibrating frequencies and collapse mechanisms can be activated more easily than in-plane ones. As well known, masonry behavior is nonlinear even at low stress levels, hence the proposed model is also able to account for material nonlinearity, by assuming a Mohr-Coulomb yield criterion for restraining interface actions [11], as it has been done by authors also with a combined finite-discrete element code [12]. However, this aspect is not investigated in this work.

Numerical tests on rectangular masonry specimens with several types of random and regular arrangements ('running' and 'stack' bond) are performed, by also considering blocks arranged with intermediate dimension along panel length ('head' bond). Modal analyses are carried on in order to evaluate the influence of blocks arrangement on frequencies and modal shapes of masonry panels. Furthermore, a homogenized 2D Reissner-Mindlin plate model identified from the discrete system is adopted for comparing results obtained with the discrete model. Moreover, the heterogeneity parameter represented by block standard size with respect to specimen dimensions is increased and, as expected, when the number of heterogeneities in the structure is large enough, the average response of the random discrete model converges to an asymptotic response. 


\section{DISCRETE RANDOM MODEL}

\subsection{Geometric random model}

In order to describe the random model, a regular one following the standard 'running bond' texture is considered for first. It is characterized by a generic block $B^{i, j}$ in contact with six surrounding blocks by means of six interfaces, where $j$ can assume any integer value and $i$ is such as $i+j$ is always even. Neighboring blocks and the corresponding interfaces are identified as $B^{i+k_{1}, j+k_{2}}$ and $S^{k_{1}, k_{2}}$, respectively, with coefficients $k_{1}, k_{2}$ defined as follows in clockwise direction (Fig. 1a):

$$
\left(k_{1}, k_{2}\right) \in\{(-1,-1),(+1,-1),(+2,0),(+1,+1),(-1,+1),(-2,0)\}
$$

Block dimensions are: width $b$, height $a$, and thickness $t$. Then, horizontal interface length is equal to block half width $b / 2$ and vertical interface length is equal to block height $a$. A 3D Cartesian coordinate system $y_{1} y_{2} y_{3}$ is introduced. Blocks are considered as rigid bodies, whereas the elastic and inelastic behavior of the system is lumped at interface or joint level, with dry or mortar joints modeled as elastic-plastic interfaces and considering plane stress hypothesis.

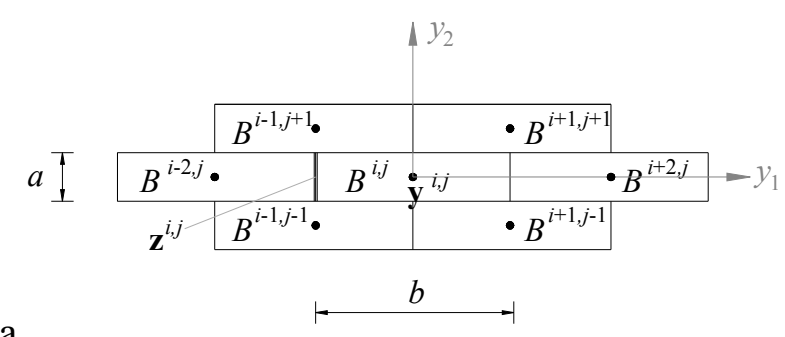

$\mathrm{a}$

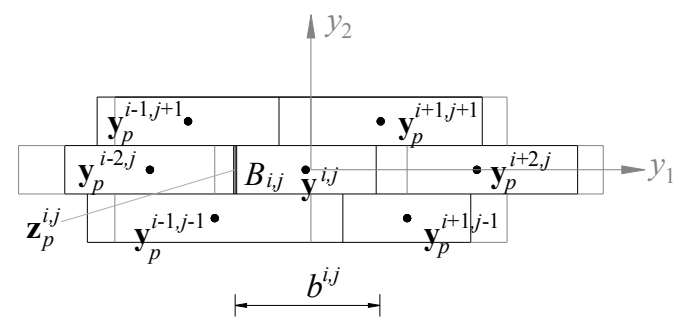

Figure 1: Representative elementary volumes (REVs) for masonry: (a) regular REV; (b) REV with perturbed block width.

The random discrete model introduced by Cecchi and Sab [6] is adopted, then all blocks keep the same height $a$, whereas block width $b$ is subject to the effect of perturbation parameters that modify the horizontal position of vertical interfaces, but that keep six surrounding blocks around the generic one. The position of $B^{i, j}$ center is $\mathbf{y}^{i, j}=i(b / 2) \mathbf{e}_{1}+j a \mathbf{e}_{2}$, then, the coordinates of the center of a vertical interface between two adjacent blocks $B^{i-2, j}$ and $B^{i, j}$ are: $\mathbf{z}^{i, j}=\left(i+k_{1} / 2\right)(b / 2) \mathbf{e}_{1}+\left(j+k_{2} / 2\right) a \mathbf{e}_{2}$. The random model is introduced by defining a random parameter in horizontal direction $p$ in order to vary the horizontal position of vertical interfaces between two adjacent blocks (Fig. 1b) as follows:

$$
\mathbf{z}_{p}^{i, j}=\mathbf{z}^{i, j}+p_{1}\left(1 / 2-X^{i, j}\right)(b / 2) \mathbf{e}_{1} .
$$

where $X^{i, j}$ is the realization of a uniform random variable defined on [0 1] and $0 \leq p<1$. 
Then, the new horizontal positions of two consecutive vertical interfaces, $\mathbf{z}_{p}^{i, j}$ and $\mathbf{z}_{p}^{i+2, j}$, allow to define the position of the new block center $\mathbf{y}_{p}^{i, j}$ and the new block width $b^{i, j}$. It is worth noting that the random model chosen maintains the original block contact topology, characterized by six neighboring blocks around $B^{i, j}$, by simply varying the length of the four horizontal contact surfaces around the block. Furthermore, the sum of the new block width along a generic course $j$ still gives the initial width of the masonry assemblage: $\Sigma_{i} b^{i, j}=L^{j}=L$ (see the following Fig. 2b, e with panels having random arrangement).

The displacement of a generic block with a random position is given by the block centre translation and by the block rigid rotation with respect to its centre as follows:

$$
\mathbf{u}^{i, j}(\mathbf{y})=\mathbf{u}^{i, j}+\mathbf{\Omega}^{i, j}\left(\mathbf{y}-\mathbf{y}_{p}^{i, j}\right)=\left\{\begin{array}{l}
u_{1}^{i, j} \\
u_{2}^{i, j} \\
u_{3}^{i, j}
\end{array}\right\}+\left[\begin{array}{ccc}
0 & -\omega_{3}^{i, j} & \omega_{2}^{i, j} \\
\omega_{3}^{i, j} & 0 & -\omega_{1}^{i, j} \\
-\omega_{2}^{i, j} & \omega_{1}^{i, j} & 0
\end{array}\right]\left\{\begin{array}{l}
y_{1}-y_{1, p}^{i, j} \\
y_{2}-y_{2, p}^{i, j} \\
y_{3}-y_{3, p}^{i, j}
\end{array}\right\} .
$$

Vector $\mathbf{q}^{i, j}=\left\{u_{1}^{i, j} u_{2}^{i, j} u_{3}^{i, j} \omega_{1}^{i, j} \omega_{2}^{i, j} \omega_{3}^{i, j}\right\}^{T}$ collects displacement components of $B^{i, j}$, given by three translations and three rotations with respect to the coordinate system.

\subsection{Mechanic model}

As previously stated, model deformability and damage are considered at interface level only. Interfaces are assumed to have an elastic-perfectly plastic behavior. Following the notation of previous authors contributions, each interface may be identified by integers $k_{1}, k_{2}$, connecting blocks $B^{i, j}$ and $B^{i+k_{1}, j+k_{2}}$ [10]. Elastic behavior is given by interface stiffness that allows to define interface actions as function of block relative displacements. For instance, relative displacements $\mathbf{d}^{k_{1}, k_{2}}=\left\{d_{1}^{k_{1}, k_{2}} d_{2}^{k_{1}, k_{2}} d_{3}^{k_{1}, k_{2}} \delta_{1}^{k_{1}, k_{2}} \delta_{2}^{k_{1}, k_{2}} \delta_{3}^{k_{1}, k_{2}}\right\}^{T}$ are three relative translations and three relative rotations between two adjacent blocks, that may be defined as functions of global block displacements: $\mathbf{d}^{k_{1}, k_{2}}=\mathbf{H}^{k_{1}, k_{2}} \mathbf{q}^{k_{1}, k_{2}}$, by means of a 'compatibility matrix' $\mathbf{H}^{k_{1}, k_{2}}$ [13]. This matrix collects distances between block centers as follows:

$$
\mathbf{H}^{k_{1}, k_{2}}=\left[\begin{array}{cccc}
-\mathbf{I} & \mathbf{D} & \mathbf{I} & \mathbf{D} \\
\mathbf{0} & -\mathbf{I} & \mathbf{0} & \mathbf{I}
\end{array}\right], \quad \mathbf{D}=\frac{1}{2}\left[\begin{array}{ccc}
0 & 0 & d y_{2} \\
0 & 0 & -d y_{1} \\
-d y_{2} & d y_{1} & 0
\end{array}\right] .
$$

Where $\mathbf{I}$ is the $3 \times 3$ identity matrix and $\mathbf{D}$ varies for each couple of adjacent blocks, accounting for the random block positions. Interface actions $\mathbf{f}^{k_{1}, k_{2}}=\left\{f_{1}^{k_{1}, k_{2}} f_{2}^{k_{1}, k_{2}} f_{3}^{k_{1}, k_{2}} m_{1}^{k_{1}, k_{2}} m_{2}^{k_{1}, k_{2}} m_{3}^{k_{1}, k_{2}}\right\}^{T}$ are obtained by integrating interface normal and shear stresses over the contact area $S^{k_{1}, k_{2}}$, leading to a normal and two shear forces and to a torsion and two bending moments, depending on interface orientation. For instance, in case of a vertical interface, normal force is $f_{2}=f_{n}$ and shear forces are $f_{1}$ and $f_{3}$, leading to an overall shear action $f_{s}^{2}=\left(f_{1}^{2}+f_{3}^{2}\right)$; moreover, bending moments are $m_{1}, m_{3}$, and torsion is $m_{2}$. Interface elastic behavior is represented by interface normal and shear stiffness $k_{n}$ and $k_{s}$, that characterize the linear relationship between interface relative displacements and actions: $\mathbf{f}^{k_{1}, k_{2}}=\overline{\mathbf{K}}^{k_{1}, k_{2}} \mathbf{d}^{k_{1}, k_{2}}=\left(\mathbf{K}^{k_{1}, k_{2}} \mathbf{A}^{k_{1}, k_{2}}\right) \mathbf{d}^{k_{1}, k_{2}}$, with $\mathbf{K}=\operatorname{diag}\left\{k_{s} k_{n} k_{s} k_{n} k_{s} k_{n}\right\}$ and $\mathbf{A}=\operatorname{diag}\left\{S S S I_{1} I_{2} I_{3}\right\}$ for a horizontal interface. Such stiffness values may be related to actual mortar elastic modulus and Poisson ratio $E^{M}, v^{M}$, together with joint thickness $e$ in case of mortar joints [10], whereas in case of dry joints, a fictitious 
mortar material may be defined. Furthermore, interface stiffness values account also for contact surface area, moment of inertia and polar inertia. Assembling interface matrices in terms of block displacements by means of compatibility matrices, the stiffness matrix of the whole masonry assemblage is obtained and the problem of a masonry panel subject to external forces $\mathbf{F}^{\text {ext }}$ may be statically solved: $\mathbf{K}^{\text {panel }} \mathbf{q}^{\text {panel }}=\mathbf{F}^{\text {ext }}$.

Interface plastic behavior is activated when actions reach their corresponding elastic limits, that are based on a tension strength and Mohr-Coulomb yield criterion and, in particular, that account for possible combined effects. All these aspects and further details related to interface elastic limits may be found in the work of Orduña and Lourenço [14] and in a recent contribution proposed by authors [11]. When actions reach the corresponding elastic limits, the corresponding stiffness values are set equal to zero. Then, interface stiffness matrix and the stiffness matrix of the whole masonry assemblage are updated together with interface actions and an incremental analysis accounting for decreasing structural stiffness can be performed, following the common procedures adopted for nonlinear analysis with finite elements accounting for material nonlinearity.

\subsection{Dynamic and modal analysis}

The equation of motion of the discrete system representing a masonry panel or assemblage is given as follows:

$$
\mathbf{M}^{\text {panel }}\left(\partial^{2} \mathbf{q} / \partial t^{2}\right)+\mathbf{K}^{\text {panel }} \mathbf{q}^{\text {panel }}=\mathbf{F}^{\text {ext }} .
$$

Where $\mathbf{M}^{\text {panel }}$ is the mass matrix of the panel collecting block mass and polar inertias with respect to coordinate axis. Assuming $\rho$ for the volumetric weight of the blocks, in this case mass and inertias have to account for the random masonry pattern, hence to the random block width $b^{i, j}$. For instance, block mass is $m^{i, j}=\rho\left(b^{i, j} a t\right)$ and block polar inertias are $J_{1}=m^{i, j}\left(a^{2}+t^{2}\right) / 12, J_{2}=m^{i, j}\left[\left(b^{i, j}\right)^{2}+t^{2}\right] / 12, J_{3}=m^{i, j}\left[\left(b^{i, j}\right)^{2}+a^{2}\right] / 12$.

In case of a large number of degrees of freedom involved in the problem due to a large number of blocks, a dynamic solution with statically applied loads (or dynamic loads) should be preferred instead of determining the stiffness matrix of the assemblage. Moreover, setting $\mathbf{F}^{\text {ext }}$ $=\mathbf{0}$, a standard eigenvalue problem is obtained $\left(\operatorname{det}\left[\mathbf{K}^{\text {panel }}-\eta^{2} \mathbf{M}^{\text {panel }}\right]=0\right)$ and the vibrating frequencies $\lambda_{i}=\eta_{i} /(2 \pi)$ of the assemblage are determined, together with the corresponding modal shapes and mass participation factors. It is worth noting that common discrete model codes are not able to perform modal analysis, since they do not determine the stiffness matrix of the discrete assemblage.

\section{HOMOGENIZED REISSNER-MINDLIN PLATE MODEL}

Discrete Element model is compared with a homogenized plate model, in which masonry is described as an equivalent continuum obtained by means of analytical homogenization procedure. The hypothesis of rigid blocks connected by elastic mortar interfaces is assumed. An orthotropic Reissner-Mindlin plate is adopted in order to account for the shear effects in the continuous model equivalent to masonry $[10,15]$. The better performance of a ReissnerMindlin plate with respect to a Love-Kirchhoff plate has been already investigated by authors for simply supported plates equivalent to masonry panels [16].

The homogenized model is not able to represent a casual random pattern, but it is able to account for a fixed perturbation parameter over the entire specimen. For instance, it is able to model a 'stack bond' pattern with aligned vertical joints [6], that may be obtained with the random discrete model by setting, for instance, $p=0.99$, and that is expected to represent a 
lower bound of the random behavior, due to its larger out-of-plane deformability given by a very small engagement between blocks.

Four masonry patterns are considered: running bond, stack bond, head running bond and head stack bond. The last two cases are characterized by an actual block width equal to one half of original width $b$. Flexural homogenized constants for running bond $D_{i j k l}^{R}, F_{i j}^{R}$ and for stack bond $D_{i j k l}^{S}, F_{i j}^{S}$, where $i, j, k, l=1,2$, are obtained in an analytical form with reference to the works $[10,17,18]$. Assuming $K^{\prime}$ and $K^{\prime \prime}$ as the bulk and shear moduli of mortar joints, the analytical expressions of homogenized moduli in the case of running bond and head running bond are:

$$
\begin{gathered}
D_{1111}^{R}=\frac{t}{12} \frac{\left[4 K^{\prime} \frac{e^{v}}{a}+\frac{b}{a} K^{\prime \prime} \frac{e^{v}}{a}\right] t^{2}+\frac{b}{4 a} \frac{e^{v}}{a} K^{\prime \prime} b^{2}}{4 \frac{e^{h}}{a} \frac{e^{v}}{b}}, \quad D_{1122}^{R}=0, \\
D_{2222}^{R}=\frac{t^{3}}{12} \frac{K^{\prime}}{\frac{e^{h}}{a}}, \quad D_{1212}^{R}=\frac{t}{192} \frac{K^{\prime \prime}\left[4 \frac{e^{h}}{a}\left(a^{2}+t^{2}\right)+4 \frac{e^{v}}{b}\left(\frac{b^{2}}{4}+t^{2}\right]+K^{\prime} \frac{b}{a} \frac{e^{v}}{a} t^{2}\right.}{\frac{e^{h}}{a} \frac{e^{v}}{b}}, \\
F_{11}^{R}=\frac{K^{\prime \prime} b t}{e^{v}}+\frac{K^{\prime \prime} b^{2} t}{a e^{h}}, \quad F_{22}^{R}=\frac{K^{\prime \prime} a t}{e^{h} .}
\end{gathered}
$$

While in case of stack bond and head stack bond are defined as:

$$
\begin{gathered}
D_{1111}^{S}=\frac{t^{3}}{12} \frac{K^{\prime}}{\frac{e^{v}}{b}}, \quad D_{1122}^{S}=0 \\
D_{2222}^{S}=\frac{t^{3}}{12} \frac{K^{\prime}}{\frac{e^{h}}{a}}, \quad D_{1212}^{S}=\frac{t}{48} K^{\prime \prime} \frac{\frac{e^{h}}{a}\left(a^{2}+t^{2}\right)+\frac{e^{v}}{b}\left(b^{2}+t^{2}\right)}{\frac{e^{h}}{a} \frac{e^{v}}{b}} \\
F_{11}^{S}=\frac{K^{\prime \prime} b t}{e^{v}}, \quad F_{22}^{S}=\frac{K^{\prime \prime} a t}{e^{h}} .
\end{gathered}
$$

\section{NUMERICAL TESTS}

A set of numerical tests is performed in order to evaluate the effectiveness of the random discrete model in representing the behavior of masonry panels with random texture, with respect to several regular cases. Random block arrangement is considered by applying the randomness in vertical interface positions, starting from a 'running bond' pattern, characterized by the following block dimensions: $b=250 \mathrm{~mm}, a=55 \mathrm{~mm}, s=120 \mathrm{~mm}$, and with a density $\rho=1800 \mathrm{~kg} / \mathrm{m}^{3}$. Joint thickness is assumed equal to $1 \mathrm{~mm}$ both for horizontal and vertical interfaces, and mortar elastic modulus and Poisson ratio are $E^{M}=1 \mathrm{GPa}$ and $v^{M}=0.2$. A rectangular masonry panel having $n_{1}=6$ blocks along horizontal direction and $n_{2}=16$ blocks along vertical direction is considered (Fig. 2a). Then, panel width $L$ is equal to $1.56 \mathrm{~m}$, whereas 
panel height $H$ turns out to be equal to $1.04 \mathrm{~m}$. Assuming these overall dimensions fixed, a 'head running bond' pattern is also considered, with $n_{1}=12$ blocks having $b=120 \mathrm{~mm}$ (Fig. $2 d)$. Starting from these regular cases, two generic random patterns are then introduced by varying vertical interface positions with a random parameter $p$ for each interface (Fig. $2 \mathrm{~b}$ and e shows two specimens generated casually); then, assuming $p=0.99$ for all interfaces, panels with vertically aligned interfaces ('stack bond') are also considered (Fig. 2c and f).

Focusing on the actual random cases (Fig. $2 \mathrm{~b}$ and e), it must be pointed out that at least ten different specimens are casually generated and studied, in order to evaluate the average effect of the randomness, but also to highlight the cases with larger differences with respect to the regular ones.

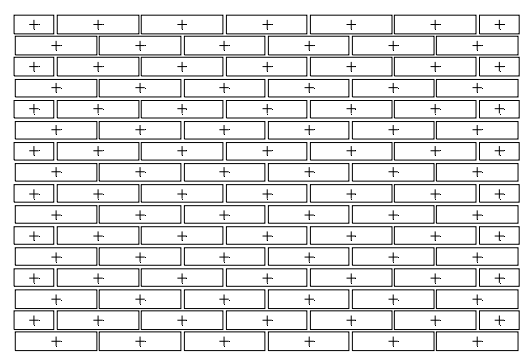

a

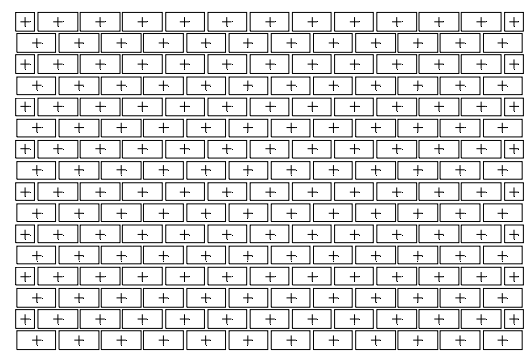

d

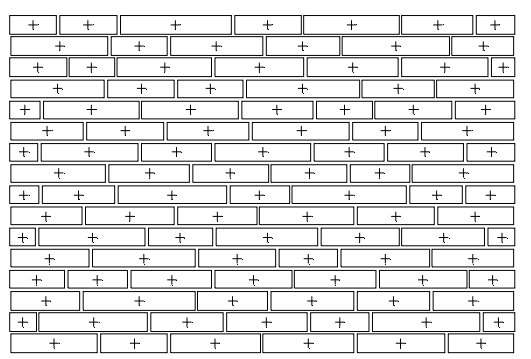

b

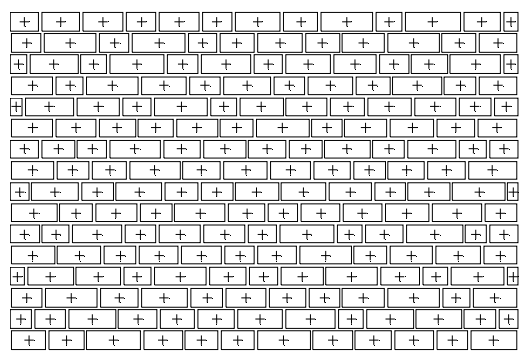

e

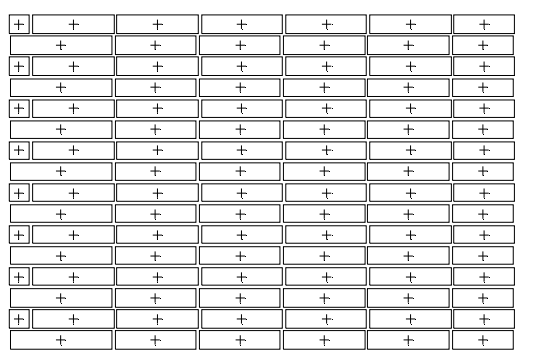

C

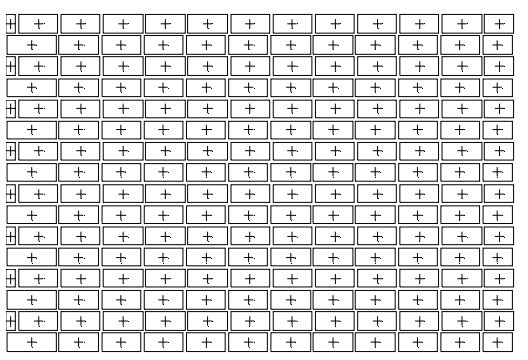

f

Figure 2: Rectangular masonry panel having (a) running bond, (b) random running bond, (c) stack bond, (d) head running bond, (e) random head bond, (f) head stack bond.

\subsection{Modal analysis with discrete model}

Modal analyses are performed on the six masonry panel types showed in Fig. 2 and having fixed base. Results obtained with the discrete model are showed in Figs. 3 and 5 in terms of modal shapes and frequencies. First and second modal shapes are always a flexural out-ofplane one in vertical direction and a torsional one, independently on blocks arrangement. The third modal shapes are flexural out-of-plane with two half-waves only for specimens obtained from the running bond texture and, similarly, the fourth modal shapes are characterized only by an in-plane flexural deformation. Considering specimens with regular and random head bond texture, the third modal shapes are flexural with a double-curved shape and the fourth modal shapes are flexural with two half waves in vertical direction. It is worth noting that the frequencies of the flexural modal shapes, with one and two half-waves, are not affected by random arrangement, given that a flexural out-of-plane mode involves only the deformation of horizontal interfaces, together with the interface stiffness in vertical direction, that depends on vertical relative distances between blocks. On the other hand, the torsional modal shapes are strictly affected by block arrangement and block size and, more generally, by the number and the position of vertical interfaces. The head stack bond case (Fig. 2f) is characterized by the lowest frequency, with a value about 34\% smaller than the frequency of the running bond case, whereas the running stack bond case (Fig. 2c) is characterized by a reduction close to 
$14 \%$. For instance, the effect of the decreasing blocks engagement starting from the running bond case, increasing the perturbation parameter $p$, is showed in Fig. 4 for torsional frequency, together with similar information for the in-plane flexural mode, characterized by frequencies at least $4 \%$ smaller than those of the regular case.

However, both stack bond cases represent lower bounds that are never reached by any one of the random specimens generated casually; in fact, average frequencies obtained with random running and head bond textures are slightly smaller than the corresponding ones obtained with the regular textures, as it is showed by the values in the second column of Figs. 3 and 5, that are closer to the values of the first column, rather than those of the third one, with values at least $1-2 \%$ smaller than those obtained with regular textures.

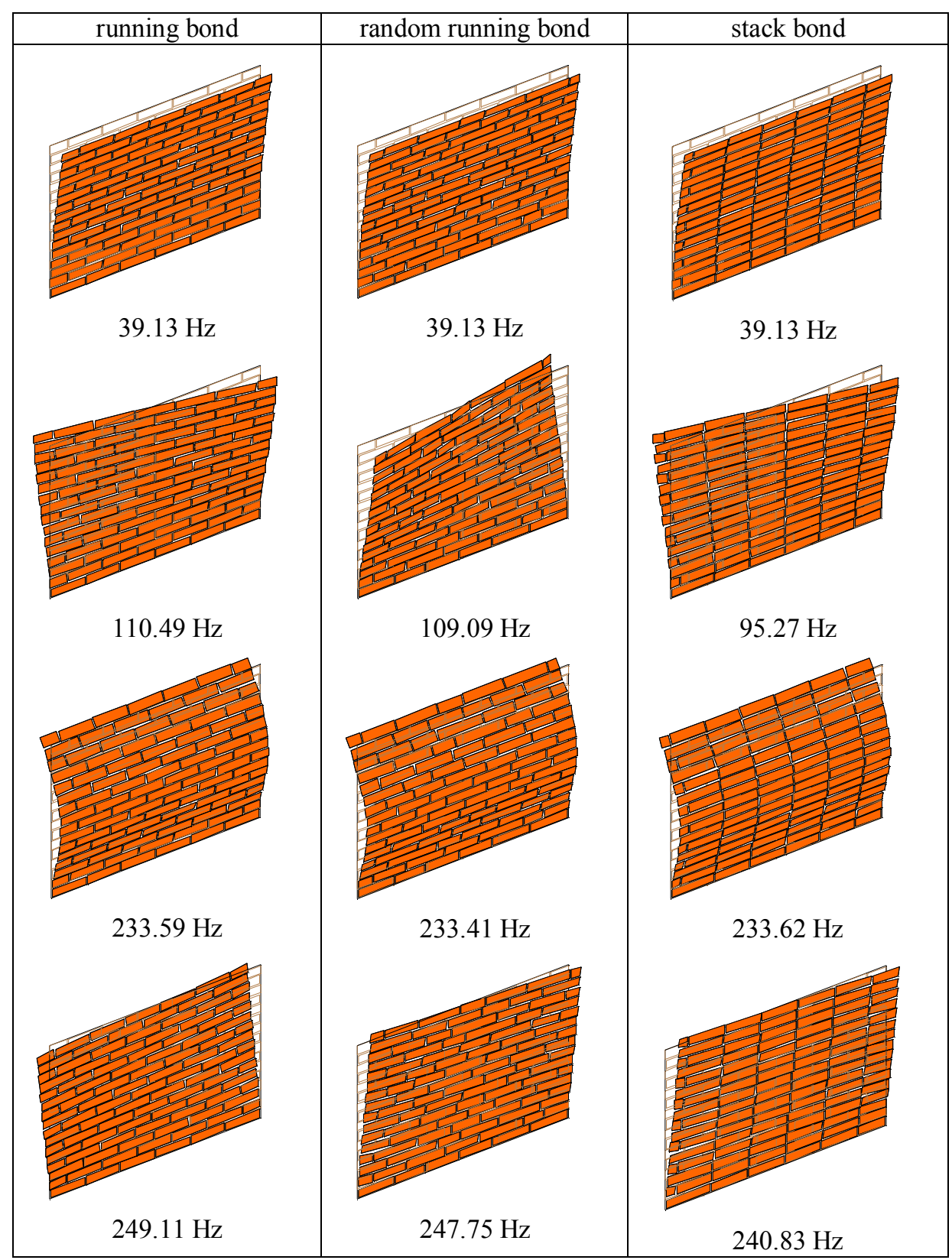

Figure 3: First four modal shapes and corresponding frequencies for masonry panels with regular and random textures obtained starting from the 'running bond' one. Block thickness is not represented for simplicity. 


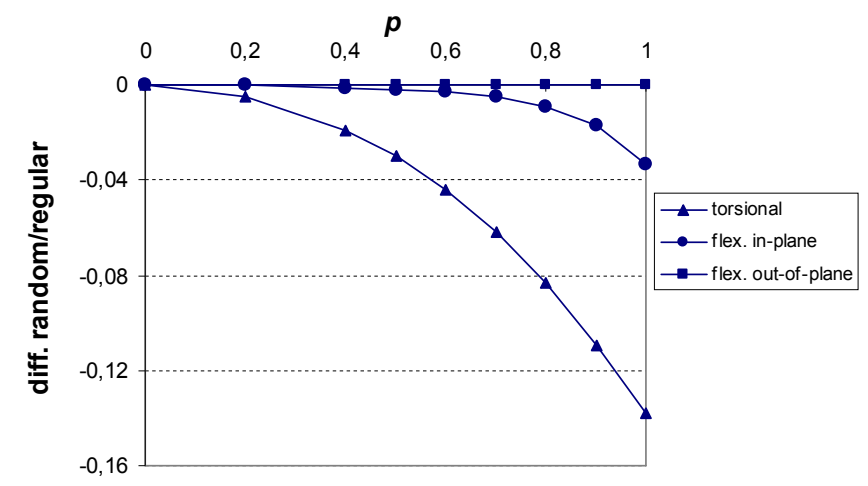

Figure 4: Differences between frequencies related to the first three modal shapes for specimens starting from a running bond regular texture and increasing the perturbation parameter.

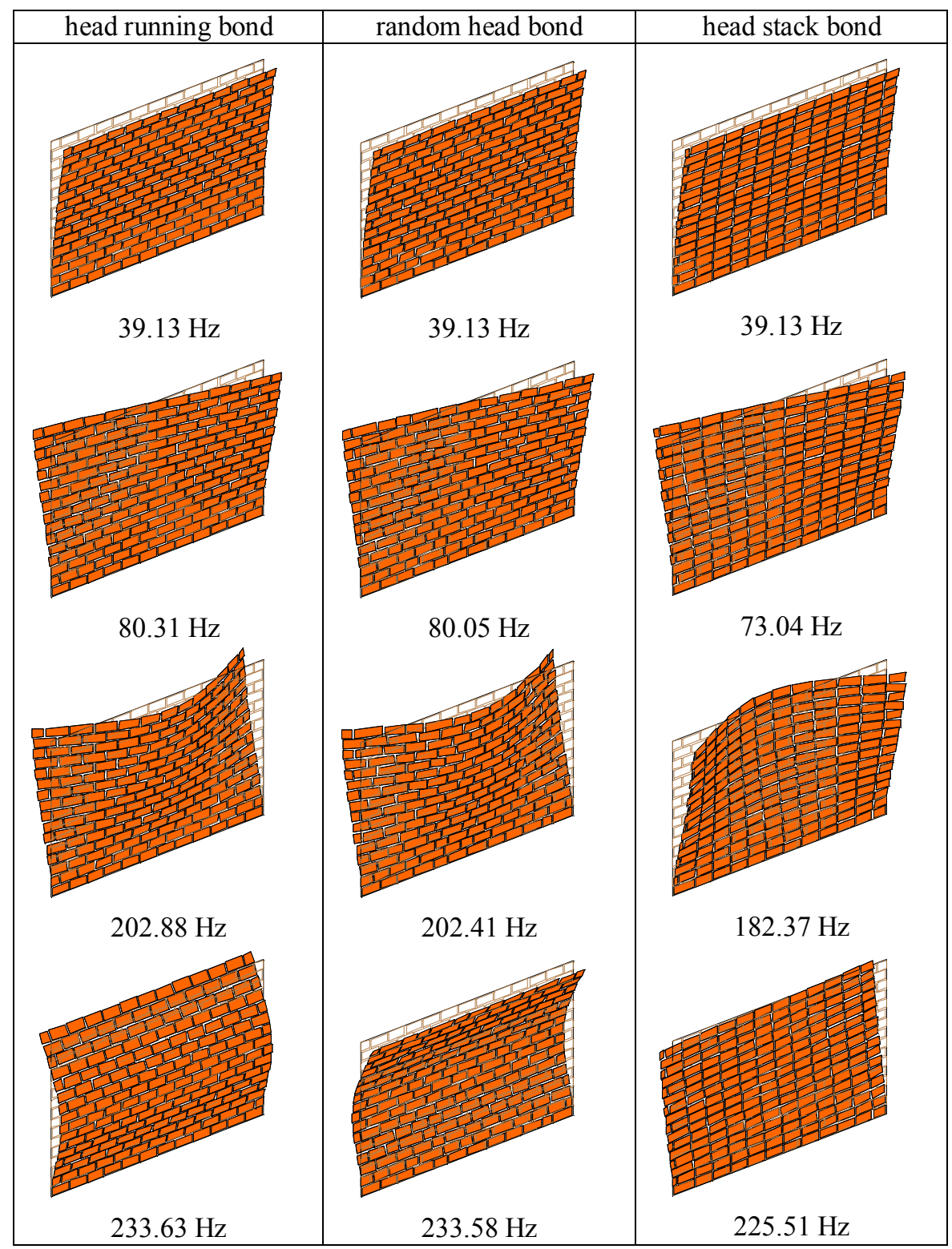

Figure 5: First four modal shapes and corresponding frequencies for masonry panels with regular and random textures obtained starting from the 'head bond' one. Block thickness is not represented for simplicity. 


\subsection{Modal analysis with homogenized model}

Modal analysis are carried on also by means of a homogenized Reissner-Mindlin plate model. Panels are modeled with quadrilateral shell elements. The use of a FE model is justified by the fact that analytical solutions in closed form for modal analysis is available only in the specific case of plate supported on the four sides [16]. Running bond, stack bond, head running bond and head stack bond patterns are considered and the corresponding homogenized flexural and shear moduli are listed in Table 1, whereas Fig. 6 shows the modal shapes and the relative frequencies obtained for the four patterns and the rectangular panel (1.66 $\mathrm{x}$ 1.04) $\mathrm{m}^{2}$ already studied with the discrete model.

As in the case of DEM, first and second modal shapes are always a flexural out-of-plane one in vertical direction and a torsional one, independently on blocks arrangement. Instead, looking at subsequent modal shapes, head bond and head stack bond patterns are characterized by a third double-curved flexural modal shape with a smaller frequency with respect to the flexural modal shape with two half-waves, that assumes the same value for the four patterns considered and appears as the third modal shape for running and stack running bond cases. The in-plane flexural modal shape appears as the fourth one for the running bond case only.

\begin{tabular}{c|rrrr}
\hline $\begin{array}{c}\text { homogenized } \\
\text { flexural and shear moduli }\end{array}$ & $\begin{array}{r}\text { running } \\
\text { bond }\end{array}$ & $\begin{array}{r}\text { stack } \\
\text { bond }\end{array}$ & $\begin{array}{r}\text { head } \\
\text { running bond }\end{array}$ & $\begin{array}{r}\text { head } \\
\text { stack bond }\end{array}$ \\
\hline$D_{1111}$ & 50730 & 26050 & 15910 & 12500 \\
$D_{1122}$ & 0 & 0 & 0 & 0 \\
$D_{2222}$ & 5731 & 5731 & 5731 & 5731 \\
$D_{1212}$ & 11750 & 3757 & 3934 & 3134 \\
\hline$F_{11}$ & 22250 & 10420 & 7727 & 5000 \\
$F_{22}$ & 2292 & 2292 & 2292 & 2292 \\
\hline
\end{tabular}

Table 1: Elastic parameters of the homogeneous material equivalent to the four masonry patterns.

The scatter between frequencies obtained with homogenized and discrete models is evaluated for increasing the number of heterogeneities $n_{1}=L / b$, keeping fixed panel and block inplane dimension ratios, together with panel and block thickness. Attention is paid to the first flexural- and second -torsional- modal shapes (Figs. 7 and 8). With a small number of blocks $n_{1}=3$, the homogenized model is not able to follow the behavior of the discrete one, and differences are generally large, close to $20-30 \%$ or more for all the patterns considered and both modal shapes. Increasing the number of blocks, differences generally decrease; however, the flexural modal shapes are all characterized by differences close to $15 \%$, independently on the masonry pattern, i.e. block engagement, given that such a modal shape is mainly governed by horizontal interfaces and, consequently, by $D_{2222}$ homogenized modulus that is the same for the four cases considered. Torsional modal shapes turn out to be very close to those obtained with the discrete model if small blocks typical of head and head stack bond patterns are modeled, given that differences are close to $8-9 \%$, but also in case of a running bond pattern. Stack bond pattern, instead, is characterized by frequencies quite far from the corresponding ones obtained with discrete models.

It is worth noting that, increasing the number of blocks, the computational effort needed for performing analysis with the homogeneous model do not vary, since the same mesh refinement should be adopted, whereas the discrete model is characterized by an increasing computational effort that limits the maximum number of blocks that can be considered and may require to consider out-of-plane degrees of freedom independently from the in-plane ones. 


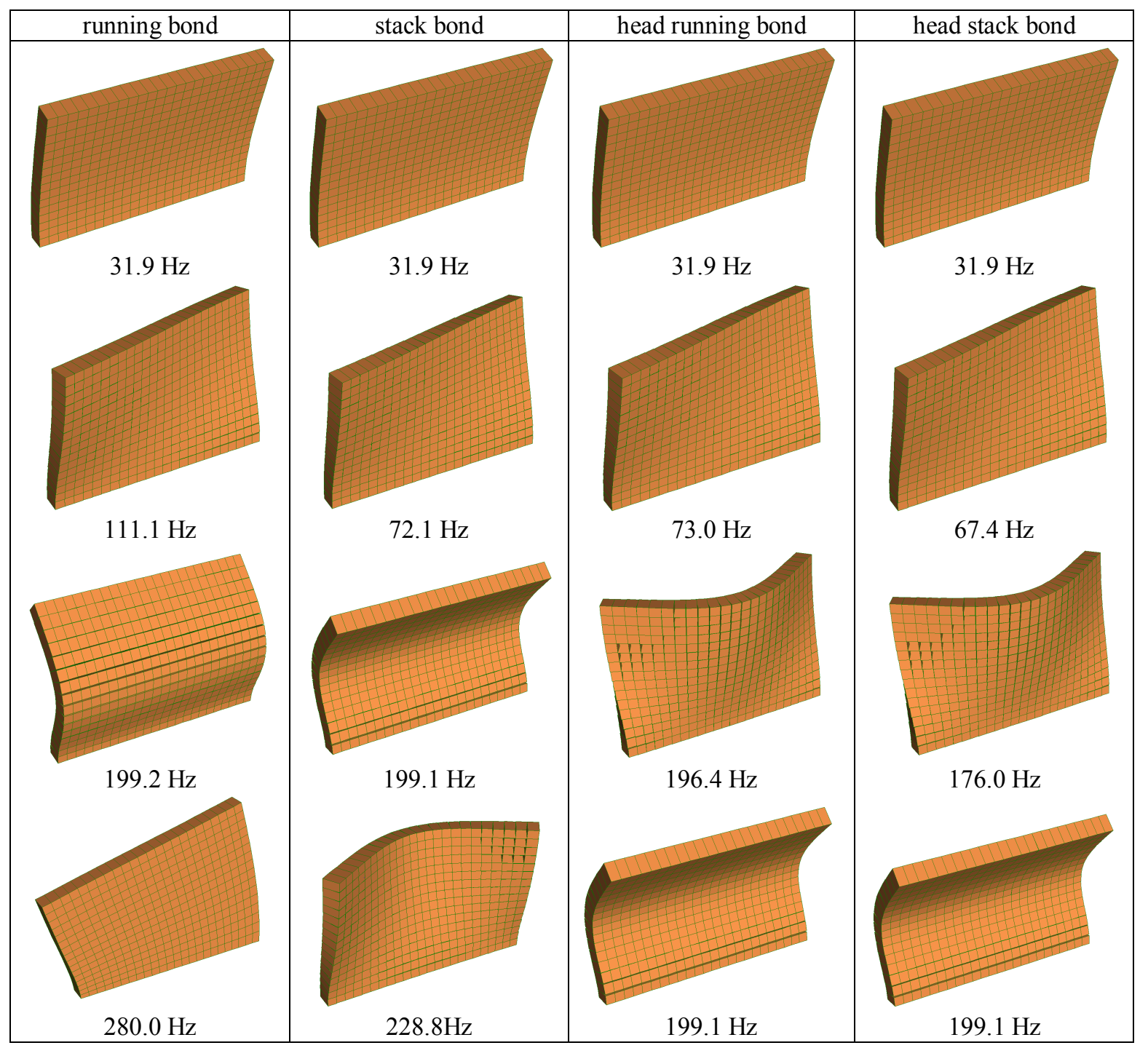

Figure 6: First four modal shapes and corresponding frequencies for masonry panels different textures modelled with homogenized plate finite elements.
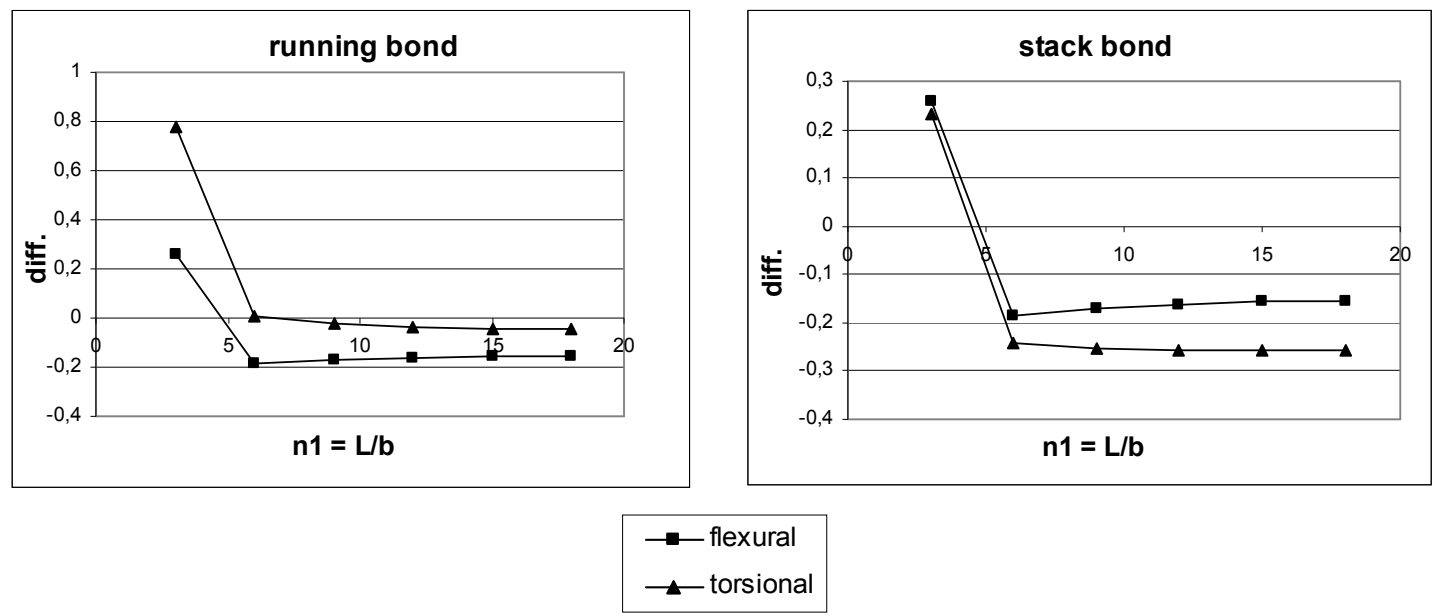

Figure 7: Differences for the first (squares) and the second (triangles) frequencies obtained with the continuous model, with respect to discrete model results, increasing the number of heterogeneities. 

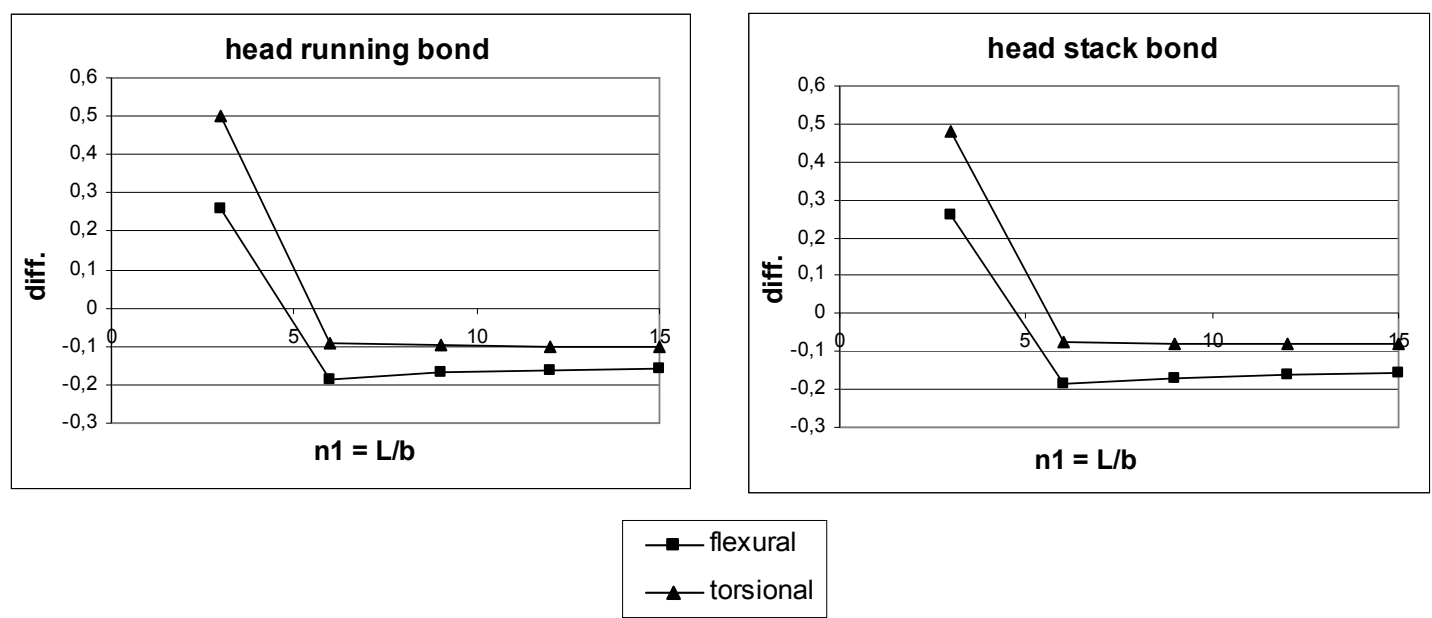

Figure 8: Differences for the first (squares) and the second (triangles) frequencies obtained with the continuous model, with respect to discrete model results, increasing the number of heterogeneities.

\section{CONCLUSIONS}

- The proposed discrete model turns out to be fast, simple and effective for performing modal analysis of masonry specimens characterized by quasi-periodic patterns with random position of vertical interfaces. The discrete model is able to generate automatically a set of casual random patterns in order to evaluate the average effects on the overall behavior of masonry panels; moreover, the matrix solution approach allows to perform modal analysis that are usually not performed by common discrete model codes.

- Considering masonry panels modeled with discrete elements, the random position of vertical interfaces do not influence significantly out-of-plane frequency and modal shapes with respect to the regular cases; only stack bond textures are characterized by a large torsional deformability that leads to decreasing frequencies for increasing the perturbation parameter $p$.

- Homogenized panels modeled with Reissner-Mindlin plate elements turn out to slightly converge to the corresponding discrete ones for increasing the number of heterogeneities. As expected, flexural modal shapes turn out to be not influenced by random patterns. However, only head bond and head stack bond patterns turn out to have the same torsional behavior of the discrete model, thanks to the larger number of blocks.

- The homogenized model is able to account for the different block arrangement in bending and shear stiffness parameters, but it is unable to account for the local torsional inertia during the determination of the mass matrix. For this aspect, homogenized model converges to the discrete one more slowly with respect to simple static analysis, where only stiffness parameters are involved [16].

- The discrete model is stiffer than the homogenized one and it is more suitable for studying masonry with actual thin joints with respect to block dimensions. However, for increasing panel size, discrete model is characterized by an increasing number of degrees of freedom that require a larger computational effort with respect to the homogenized model, that turns out to be simpler and faster, given that the same FE mesh refinement may be adopted for increasing panel size.

- Further developments of this work will regard pushover and dynamic analysis of specimens with random texture, in order to evaluate the influence of randomness on collapse 
mechanisms and ultimate loads, also accounting for randomness in vertical direction by varying the height of each course of blocks, as it has been recently done by authors for the in-plane case [19].

- Further developments of this work will regard the study of actual 3D specimens such as small buildings or building portions with orthogonal walls, slabs, roofs and openings.

\section{ACKNOWLEDGMENTS}

The research has been carried out thanks to the financial support of PRIN 2015 (under grant 2015JW9NJT_014, project "Advanced mechanical modeling of new materials and structures for the solution of 2020 Horizon challenges").

\section{REFERENCES}

[1] T.M. Ferreira, A.A. Costa, A. Costa, Analysis of the Out-Of-Plane Seismic Behavior of Unreinforced Masonry: A Literature Review. International Journal of Architectural Heritage, 9(8), 949-972, 2015.

[2] H. Smoljanović, N. Živaljić, Ž.Nikolić, Overview of the methods for the modeling of historical masonry structures. Gradevinar, 65(7), 607-618, 2013.

[3] F. Cluni, V. Gusella, Homogenization of non-periodic masonry structures, International Journal of Solids and Structures, 41(7) 1911-1923, 2004.

[4] N. Cavalagli, F. Cluni, V. Gusella, Strength domain of nonperiodic masonry by homogenization in generalized plane state, European Journal of Mechanics A/Solids, 30, 113126, 2011.

[5] G. Falsone, M. Lombardo, Stochastic representation of the mechanical properties of irregular masonry structures, International Journal of Solids and Structures, 44(25-26) 8600-8612, 2007.

[6] A. Cecchi and K. Sab, A homogenized Love-Kirchhoff model for out-of-plane loaded random 2D lattices: Application to "quasi-periodic" brickwork panels, International Journal of Solids and Structures, 46, 2907-2919, 2009.

[7] G. Milani, P.B. Lourenço, A simplified homogenized limit analysis model for randomly assembled blocks out-of-plane loaded, Computer \& Structures, 88(11-12), 690-717, 2010.

[8] G. Milani, Y.W. Esquivel, P.B. Lourenço, B. Riveiro, D.V. Oliveira, Characterization of the response of quasi-periodic masonry: Geometrical investigation, homogenization and application to the Guimarães castle, Portugal, Engineering Structures, 56, 621-641, 2013.

[9] J.V. Lemos, Discrete Element Modeling of Masonry Structures. International Journal of Architectural Heritage, 1, 190-213, 2007.

[10] A. Cecchi, K. Sab, A comparison between a 3D discrete model and two homogenised plate models for periodic elastic brickwork, International Journal of Solids and Structures, 41(9-10), 2259-2276, 2004. 
[11] D. Baraldi, A. Cecchi, A full 3D rigid block model for the collapse behaviour of masonry walls, European Journal of Mechanics A/Solids, 64, 11-28, 2017.

[12] E. Reccia, A. Cazzani, A. Cecchi, FEM-DEM Modeling for Out-of-plane Loaded Masonry Panels: A Limit Analysis Approach, Open Civil Engineering Journal, 6(1), 231238, 2012.

[13] M.C. Ferris, F. Tin-Loi, Limit analysis of frictional block assemblies as a mathematical program with complementarity constraints, International Journal of Mechanical Sciences, 43, 209-224, 2001.

[14] A. Orduña, P.B. Lourenço, Three-dimensional limit analysis of rigid blocks assemblages. Part I: Torsion failure on frictional interfaces and limit analysis formulation. International Journal of Solids and Structures, 42(18-19), 5140-5160, 2005.

[15] A. Cecchi, K. Sab, Out of plane model for heterogeneous periodic materials: The case of masonry, European Journal of Mechanics, A/Solids, 21(5), 715-746, 2002.

[16] D. Baraldi, A. Cecchi, Discrete and continuous models for static and modal analysis of out of plane loaded masonry. Computer \& Structures, 2017 (in press, doi: 10.1016/j.compstruc.2017.03.015).

[17] A. Cecchi, K. Sab, Erratum: A comparison between a 3D discrete model and two homogenised plate models for periodic elastic brickwork (International Journal of Solids and Structures (2004) 41 (2259-2276) DOI: 10.1016/j.ijsolstr.2003.12.020), International Journal of Solids and Structures, 43 (2) 390-392, 2006.

[18] A. Cecchi, K. Sab, A homogenized Reissner-Mindlin model for orthotropic periodic plates: Application to brickwork panels, International Journal of Solids and Structures, 44 (18-19), 6055-6079, 2007.

[19] D. Baraldi, A. Cecchi, Discrete model for the collapse behavior of unreinforced random masonry walls, Key Engineering Materials, 2017 (in press). 